Car bon i mpuriti es behavi or and its i mpact on i on ther mal conf i nement in

hi gh- $\mathrm{i}$ on- temper at ure deut er i um di schar ges on the Large Hel i cal Devi ce

\begin{tabular}{|l|l|}
\hline $\begin{array}{l}\text { jour nal or } \\
\text { publ i cat i on } \mathrm{ti} \text { t l e }\end{array}$ & $\mathrm{Pl}$ asma Physi cs and Cont rol I ed Fusi on \\
\hline vol une & 60 \\
\hline nunber & 7 \\
\hline page $\mathrm{r}$ ange & 074005 \\
\hline year & 2018- 05-15 \\
\hline URL & ht t p: //hdl . handl e. net /10655/00012658 \\
\hline
\end{tabular}




\title{
Carbon impurities behavior and its impact on ion thermal confinement in high-ion-temperature deuterium discharges on
} the Large Helical Device

\author{
K. Mukai ${ }^{1,2}$, K. Nagaoka ${ }^{1,3}$, H. Takahashi ${ }^{1,2}$, M. Yokoyama ${ }^{1,2}$, S. Murakami ${ }^{4}$, \\ H. Nakano ${ }^{1,2}$, K. Ida ${ }^{1,2}$, M. Yoshinuma ${ }^{1,2}$, R. Seki ${ }^{1,2}$, S. Kamio ${ }^{1}$, Y. Fujiwara ${ }^{1}$, T. Oishi ${ }^{1,2}$, \\ M. Goto ${ }^{1,2}$, S. Morita ${ }^{1,2}$, T. Morisaki ${ }^{1,2}$, M. Osakabe ${ }^{1,2}$ and the LHD Experiment Group ${ }^{1}$ \\ ${ }^{1}$ National Institute for Fusion Science, National Institutes of Natural Sciences, Toki, Gifu \\ 509-5292, Japan \\ ${ }^{2}$ SOKENDAI (Graduate University for Advanced Studies), Toki, Gifu 509-5292, Japan \\ ${ }^{3}$ Graduate School of Science, Nagoya University, Nagoya, Aichi 464-8601, Japan \\ ${ }^{4}$ Department of Nuclear Engineering, Kyoto University, Kyoto, Kyoto 615-8510, Japan \\ E-mail: mukai.kiyofumi@nifs.ac.jp
}

\begin{abstract}
The behavior of carbon impurities in deuterium plasmas and its impact on thermal confinement were investigated in comparison with hydrogen plasmas in the Large Helical Device (LHD). Deuterium plasma experiments have been started in the LHD and high-iontemperature plasmas with central ion temperature $\left(T_{\mathrm{i}}\right)$ of $10 \mathrm{keV}$ were successfully obtained. The thermal confinement improvement could be sustained for a longer time compared with the hydrogen plasmas. An isotope effect was observed in the time evolution of the carbon density profiles. A transiently peaked profile was observed in the deuterium plasmas due to the smaller carbon convection velocity and diffusivity in the deuterium plasmas compared with the hydrogen plasmas. The peaked carbon density profile was strongly correlated to the ion thermal confinement improvement. The peaking of the carbon density profile will be one of the clues to clarify the unexplained mechanisms for the formations of ion internal transport barrier and impurity hole on LHD. These results could also lead to a better understanding of the isotope effect in the thermal confinement in torus plasma.
\end{abstract}




\section{Introduction}

Understanding the impact of light impurities on plasma confinement is one of the crucial issues to realize fusion reactors not only in tokamak type but also in helical type. In the high-triangularity H-mode on JET-ILW, the pedestal pressure increased with the increase of nitrogen seeding, however, the relation is opposite in JET-C $[1,2]$. In the H-mode on JT-60U, the pedestal carbon density and the plasma effective charge $Z_{\text {eff }}$ in deuterium plasmas were higher than that in hydrogen plasmas [3]. While it is known that the light impurities play an important role on confinement, the isotope effect of the role is still unclear.

In the Large Helical Device (LHD), high ion temperature $\left(T_{\mathrm{i}}\right)$ operations with ion internal transport barrier (ITB) have been studied [4-6] and significant physics phenomena were found, e.g. break of gyroBohm dependence of thermal transport and impurity hole [7, 8]. Strong correlations between the densities of carbon impurities $n_{\mathrm{c}}$ and the ion thermal diffusivities $\chi_{\mathrm{i}}$ [9] were found in high $T_{\mathrm{i}}$ operations using hydrogen plasmas. The central $T_{\mathrm{i}}$ increased after a carbon pellet injection and $\chi_{\mathrm{i}}$ decreased with the enhanced $n_{\mathrm{c}}$ decrease. $\chi_{\mathrm{i}}$ at the normalized minor radius $r_{\mathrm{eff}} / a_{99} \sim 0.72$ was minimized when $n_{\mathrm{c}} \sim 4 \times$ $10^{17} \mathrm{~m}^{-3}$. When $n_{\mathrm{c}}$ decreased below a threshold in the formation of the impurity hole, $\chi_{\mathrm{i}}$ suddenly increased. While the impurity hole prevented the accumulation of impurities, the lack of the impurities degraded the ion thermal confinement. Deuterium plasma experiments have been started in the LHD [10]. High- $T_{\mathrm{i}}$ plasmas of $10 \mathrm{keV}$ were successfully obtained with upgraded neutral beam injection (NBI) heating and optimization of the experimental conditions. Moreover, the high- $T_{\mathrm{i}}$ deuterium plasmas could be sustained for a longer time compared with the hydrogen plasmas, which indicates the relation between $\chi_{\mathrm{i}}$ and $n_{\mathrm{c}}$ in deuterium plasmas is different from that in hydrogen plasmas. In this study, the behavior of the carbon impurities in deuterium plasmas is investigated and the comparison with hydrogen plasmas is discussed. The experimental setup is shown in section 2. The general explanation of high-iontemperature discharges is described as the comparison between hydrogen discharge and deuterium discharge in section 3. In section 4, particle transport analysis of the carbon impurities and thermal transport analysis are carried out. The relation between the thermal transport and carbon density is discussed. The paper is summarized in section 5 .

\section{Experimental setup}

The experimental setup in LHD is shown in Figure 1. LHD is a large-sized helical device. The major radius $R$ is $3.9 \mathrm{~m}$ and the averaged minor radius $a_{99}$ is $0.65 \mathrm{~m}$. The magnetic field is generated using superconducting helical coils with poloidal/toroidal period numbers $\mathrm{L} / M=2 / 10$ and three pairs of superconducting poloidal coils. In this study, the magnetic field was set with the major radius of the magnetic axis $R_{\mathrm{ax}}=3.60 \mathrm{~m}$ and the toroidal magnetic field strength $B_{\mathrm{t}}=-2.85 \mathrm{~T}$. Wall recycling was reduced as possible by discharge cleaning since the ion ITB forms with a low recycling wall condition [6]. Plasmas were generated using electron cyclotron heating $(\mathrm{ECH})$ and were sustained using NBI. NBI 
has five beam lines. NBI \#1-3 have tangential beam lines and have negative ion sources with the hydrogen beam injection energy of $\sim 180 \mathrm{keV}$ for both hydrogen plasmas and deuterium plasmas. NBI \#4-5 have radial beam lines and positive ion sources. For hydrogen plasmas, the hydrogen beam injection energy is $\sim 40 \mathrm{keV}$ each. For deuterium plasmas, the NBI \#4-5 injection energy of deuterium beam increased to $\sim 60 \mathrm{keV}$ and $\sim 80 \mathrm{keV}$, respectively. A carbon pellet was injected from the outer port at $10(10-\mathrm{O})[11,12]$. The carbon pellet has the cylindrical shape with the diameter of $1.05 \mathrm{~mm}$ and length $1.05 \mathrm{~mm}$. The $T_{\mathrm{i}}$ and $n_{\mathrm{c}}$ were measured using charge exchange spectroscopy (CXS) [13]. To evaluate the background signals of the measurement, NBI \#4 was operated with $10 \mathrm{~Hz}$ modulation. The background signals can be determined as the signal in the turned off phase.

\section{High- $T_{\mathrm{i}}$ plasmas with carbon pellet}

Waveforms of high- $T_{\mathrm{i}}$ plasmas in the hydrogen (\#123145) and deuterium (\#133707) discharges are shown in Figure 2 and Figure 3, respectively. The total port-through power of NBI with negative ion sources $(\mathrm{N}-\mathrm{NBI})$ is $\sim 16 \mathrm{MW}$ and the total power of NBI positive ion sources (P-NBI) is $\sim 10 \mathrm{MW}$ in the hydrogen plasma. In the deuterium plasma, the total port-through powers of N-NBI and P-NBI are $\sim 14 \mathrm{MW}$ and $\sim 17 \mathrm{MW}$, respectively. The difference is caused by the optimization target gas of the NBI systems mentioned in the section 2. A carbon pellet was injected at $t=4.606 \mathrm{~s}$ and $4.581 \mathrm{~s}$ for $\mathrm{H}$ and D plasmas, respectively. After the injection, plasma stored energy $W_{\mathrm{p}}$ and line-averaged electron density $\bar{n}_{\mathrm{e}}$ suddenly increased in both cases. In Fig. 3 (b), burst signals of plasma radiated power were observed after the pellet injection. The first one corresponds to the pellet injection, but the second and third burst signals are due to the events of energetic-ion-driven resistive interchange mode (EIC) [14]. The central $T_{\mathrm{i}}$ increased after the pellet injection in the both cases. Higher central $T_{\mathrm{i}}$ plasmas were obtained in the deuterium plasmas. Moreover, the higher central $T_{\mathrm{i}}$ could be sustained for longer time until the NBI break down while the central $T_{\mathrm{i}}$ in the hydrogen plasma decreased from $0.2 \mathrm{~s}$ after the carbon pellet injection. Figure 4 shows the radial profiles of $T_{\mathrm{i}}$ in the deuterium and the hydrogen discharges at the time of the maximum central ion temperature. $T_{\mathrm{i}}$ in the deuterium plasma increased compared with the hydrogen plasma case, especially $\left|r_{\text {eff }} / a_{99}\right|<0.2$ where the profile is flattened in the hydrogen plasma. As shown in Fig. 2 (d), (e) and Fig. 3 (d), (e), the electron temperature $T_{\mathrm{e}}$ and the electron density $n_{\mathrm{e}}$ profiles were not changed drastically when the central $T_{\mathrm{i}}$ reached the maximum. Since the density of the carbon pellet is $2 \mathrm{~g} \cdot \mathrm{cm}^{-3}$, when the injected carbon is fully ionized, increase of $\bar{n}_{\mathrm{e}}$ is expected $2 \times 10^{19} \mathrm{~m}^{-3}$. The observation shows the increase of $\bar{n}_{\mathrm{e}}$ just after the carbon pellet injection was approximately $1.2 \times 10^{19} \mathrm{~m}^{-3}$ in the both cases. Then some carbon particles were partially

ionized. Figure 5 shows the $\bar{n}_{\mathrm{e}}$ dependence of central $T_{\mathrm{i}}$ in the all high- $T_{\mathrm{i}}$ deuterium discharges of the experimental campaign in 2017. The plots contain all conditions e.g. various of the magnetic configuration and the heating power. The data indicates that $\bar{n}_{\mathrm{e}}=1 \sim 1.5 \times 10^{19} \mathrm{~m}^{-3}$ is optimal to produce the higher $T_{\mathrm{i}}$ plasmas. Time evolution of $n_{\mathrm{c}}$ are indicated in Fig. 2 (f) and Fig. 3 (f). The decay time of $n_{\mathrm{c}}$ in the deuterium plasma was slower compared with the hydrogen plasma. $n_{\mathrm{c}}$ profile shapes were 
obviously different. In the deuterium plasma, $n_{\mathrm{c}}$ profile was peaked from $4.7 \mathrm{~s}$ to $4.9 \mathrm{~s}$. The peaking factor shown in Fig. 2 (g) and Fig. $3(\mathrm{~g})$ was determined as the $n_{\mathrm{c}}$ ratio between at $r_{\mathrm{eff}} / a_{99} \sim 0.2$ and at $r_{\text {eff }} / a_{99} \sim 0.5 . n_{\mathrm{c}}$ profiles finally changed to a hollow shape in the both cases so-called impurity hole at $t$ $>4.9$ s. Fig. 2 (h) and Fig. 3 (h) indicates the density fraction of hydrogen, deuterium, and helium. In the hydrogen plasma, there is no deuterium since the discharge was before the deuterium experiment. Here, helium was used for wall conditioning by discharge cleaning, for main gas puffing in main discharges to maintain a low wall recycling, and for a propellant gas of the carbon pellet. While the high- $T_{\mathrm{i}}$ plasma was sustained, the density fraction of helium ions was 0.4 approximately in the both cases.

\section{Results and discussion}

\subsection{Peaking of carbon density profile in deuterium discharges}

Time evolution of the $n_{\mathrm{c}}$ profiles after the carbon pellet injection in the hydrogen and deuterium discharges is shown in Figure 6 and Figure 7, respectively. In the both cases, carbon pellet was absorbed around $r_{\mathrm{eff}} / a_{99} \sim 0.8$ and $n_{\mathrm{c}}$ profiles took hollow shapes $0.3 \mathrm{~s}$ after the injection $(t>4.9 \mathrm{~s})$. The main differences in the profiles are observed around $t=4.7 \mathrm{~s}$ as shown in Fig. 6 (b) and Fig. 7 (b). The $n_{\mathrm{c}}$ profile had a peaked shape transiently in the deuterium discharge while the profile was slightly hollow in the hydrogen discharge.

As shown in Fig. $3(\mathrm{~g})$, the peaked $n_{\mathrm{c}}$ profile in the deuterium discharge was formed by $t=4.7 \mathrm{~s}$. In order to characterize carbon impurity behaviors in the formation of the peaked $n_{\mathrm{c}}$ profile and in the relaxation process of the absorbed carbon impurities, transport analysis with a diffusion model was carried out. Carbon particle flux $\Gamma_{\mathrm{c}}$ normalized by $n_{\mathrm{c}}$ can be expressed as follows [8],

$$
\frac{\Gamma_{c}}{n_{c}}=-D_{c} \frac{\nabla n_{c}}{n_{c}}+V_{c},
$$

where $D_{\mathrm{c}}$ and $V_{\mathrm{c}}$ are diffusivity and convection velocity of carbon, respectively. $\Gamma_{\mathrm{c}}$ can be evaluated by

$$
\Gamma_{c}(r)=\frac{1}{r} \int_{0}^{r} r^{\prime}\left(S\left(r^{\prime}\right)-\frac{\mathrm{d} n_{c}\left(r^{\prime}\right)}{\mathrm{d} t}\right) \mathrm{d} r^{\prime},
$$

where $S$ is source term of carbon impurities. The main candidates of carbon impurities can be considered as the injected carbon pellet and the sputtered carbon from the divertor plate. When we consider the carbon transport after the absorption, the injected carbon is fully ionized. Moreover, the sputtered carbon cannot penetrate into the plasma core region before ionization. Therefore, $S$ is negligible for the analysis. Figure 8 shows the relation between carbon particle flux $\Gamma_{\mathrm{c}}$ and $-\nabla n_{\mathrm{c}}$ in the hydrogen discharge (a) and the deuterium plasma (b). The time range of the plots in Figure 8 is $4.62<t<4.66 \mathrm{~s}$ which means the period during the formation of the peaked $n_{\mathrm{c}}$ profile in the deuterium plasma as shown in Fig. 7 (b) and correspond to the flat-top phase of the injected power by NBI \#4. Since $\Gamma_{\mathrm{c}}$ and $-\nabla n_{\mathrm{c}}$ are normalized by $n_{\mathrm{c}}$, the slope and intercept of the linear least-squares fit in Fig. 8 correspond to $D_{\mathrm{c}}$ and $V_{\mathrm{c}}$, respectively. The radial profiles of $D_{\mathrm{c}}$ and $V_{\mathrm{c}}$ estimated by Fig. 8 are shown in Figure 9 . The plot of $r_{\mathrm{eff}} / a_{99} \sim 0.3$ in 
Fig. 8 (a) was too clustered for evaluation by fitting. In the deuterium plasma, $D_{\mathrm{c}}$ is smaller, that is, confinement is better in a wider region of minor radius. $V_{\mathrm{c}}$ is outward in both discharges; as a result an impurity hole is formed. However, the $V_{\mathrm{c}}$ in the deuterium plasma is less than half of that in the hydrogen discharge and changing to inward at $r_{\text {eff }} / a_{99} \sim 0.3$. These difference in the particle transport can be considered to cause a difference in the time evolution of $n_{\mathrm{c}}$, such as the peaked profile in the deuterium plasma as shown in Fig. 7 (b). The isotope effect of the carbon particle transport has the potential to affect on the ion thermal transport since the strong correlation between $n_{\mathrm{c}}$ and ion thermal transport was observed in hydrogen plasmas [9].

\subsection{Impact of carbon impurities on ion thermal transport}

In the previous study with hydrogen plasmas [9], the relation between $n_{\mathrm{c}}$ and thermal diffusivity $\chi_{\mathrm{i}}$ is almost identical regardless of being a single-pellet discharge or double-pellet discharge. As mentioned in the section 3, in the deuterium plasmas, higher central $T_{\mathrm{i}}$ plasmas were obtained and the higher central $T_{\mathrm{i}}$ could be sustained for longer time. It indicates the relation between $\chi_{\mathrm{i}}$ and $n_{\mathrm{c}}$ in deuterium plasmas is different from that in hydrogen plasmas. Then we discuss the relation as the comparison between hydrogen and deuterium discharges. Figure 10 shows the relation between $n_{\mathrm{c}}$ and $\chi_{\mathrm{i}}$ evaluated using the DYTRANS package of the TASK3D code [15] at $4.7<t<5.0 \mathrm{~s}$. $\bar{n}_{\mathrm{e}}$ was too high at $4.6<t<4.7 \mathrm{~s}$ for the thermal transport analysis and during the EIC events $\chi_{\mathrm{i}}$ cannot be evaluated due to the fast ion loss. Thus, these periods were removed from the analysis. Initially, $\chi_{\mathrm{i}}$ slightly decreased with the $n_{\mathrm{c}}$ decrease, but later $\chi_{\mathrm{i}}$ suddenly increased with the further $n_{\mathrm{c}}$ decrease. At $r_{\text {eff }} / a_{99} \sim 0.7$, as shown in Fig. 10 (b) the $\chi_{\mathrm{i}}$ in the deuterium plasma was slightly lower than that in the hydrogen plasma but it is not significant. However, the $\chi_{\mathrm{i}}$ at $r_{\mathrm{eff}} / a_{99} \sim 0.2$ in the deuterium plasma was $20 \%$ lower than in the hydrogen plasma when $n_{\mathrm{c}} \sim 0.8 \times 10^{17} \mathrm{~m}^{-3}$. This result corresponds to the increase of the central $T_{\mathrm{i}}$ and its sustainment as shown in Fig. 4.

Two candidates for the mechanism of the relation between $n_{\mathrm{c}}$ and $\chi_{\mathrm{i}}$ were mentioned in the previous study [9]. One is a reduction in the linear growth rate of ion temperature gradient (ITG) mode by an increase of the plasma effective charge $\left(Z_{\text {eff }}\right)$ like the radiative improve mode on TEXTOR-94 [16-18]. The other is the scaling of the linear growth rate of the ITG mode by the plasma effective mass $M_{\text {eff }}$ for tokamak devices, $\gamma_{\mathrm{ITG}} \sim M_{\mathrm{eff}}^{0.5}[19]$. Although both $Z_{\mathrm{eff}}$ and $M_{\mathrm{eff}}$ in the deuterium plasma were higher than those in the hydrogen plasma, $Z_{\text {eff }}$ and $M_{\text {eff }}$ decreased with the decrease of $n_{\mathrm{c}}$ also in the deuterium plasma. It indicates that the suddenly increase of $\chi_{\mathrm{i}}$ at low $n_{\mathrm{c}}$ cannot be explained only by these candidates. Since $Z_{\text {eff }}$ and $M_{\text {eff }}$ were evaluated with the assumption that the density fraction among hydrogen, deuterium, and helium is uniform radially, the profile measurement of the fraction will progress further investigation.

One more candidate for the mechanism of the relation between $n_{\mathrm{c}}$ and $\chi_{\mathrm{i}}$ can be proposed by the discussion in the section 4.1 . The shape of $n_{\mathrm{c}}$ profile can be changed widely using the deuterium plasmas. Figure 11 shows the $\chi_{\mathrm{i}}$ as a function of the peaking factor of the $n_{\mathrm{c}}$. This result indicates that $n_{\mathrm{c}}$ peaking is strongly correlated to the ion thermal confinement improvement. The sustainment of the higher- $T_{\mathrm{i}}$ in 
the deuterium plasma is consistent with the improvement of the ion thermal confinement due to the $n_{\mathrm{c}}$ peaking. The $n_{\mathrm{c}}$ peaking will be one of the clues to clarify the unexplained mechanisms for the formations of ion ITB and impurity hole on LHD.

\section{Summary}

In this paper, behavior of the carbon impurities and its impact on ion thermal confinement in high-iontemperature discharges were investigated by comparing deuterium plasmas and hydrogen plasmas in the LHD. The high- $T_{\mathrm{i}}$ plasmas could be sustained for a longer period using deuterium. The isotope effect was observed in the time evolution of the $n_{\mathrm{c}}$ profiles. $n_{\mathrm{c}}$ profile was transiently peaked in the deuterium plasmas during the formation phase of the impurity hole. The peaking was formed by the lower convection velocity and the lower diffusivity of the carbon impurities. While the mechanism for the ion thermal confinement improvement could not be explained by the reduction in the linear growth rate of ITG mode by the increase of the $Z_{\text {eff }}$ or $M_{\text {eff }}$, the carbon density peaking was strongly correlated to the improvement. The profile measurement of the density fraction among hydrogen, deuterium, and helium are required to obtain better conclusion about $Z_{\text {eff }}$ or $M_{\text {eff. }} n_{\mathrm{c}}$ profile control using TESPEL (tracerencapsulated solid pellet injection system) [20] or granule injection [21] is left for future study. According to the initial observation, $n_{\mathrm{c}}$ profile was changed depending on the position of the magnetic axis. These will be the clues to explore the underlying mechanisms for the formations of ion ITB and impurity hole on LHD. Since the behavior of the light impurities is important regardless of the wall material and reactor type, these results can lead to the resolution of the isotope effect in the thermal confinement improvement not only in helical plasmas but also in tokamak plasmas.

\section{Acknowledgements}

The authors thank the LHD technical staff for their excellent support in the LHD experiments. This work is supported by NIFS/NINS (NIFS16UMLG701, NIFS16ULGG801).

\section{References}

[1] Giroud C et al 2013 Nucl. Fusion 53113025

[2] Maddison G P et al 2014 Nucl. Fusion 54073016

[3] Urano H et al 2012 Nucl. Fusion 52114021

[4] Ida K et al 2010 Contrib. Plasma Phys. 50558

[5] Takahashi H et al 2013 Nucl. Fusion 53073034

[6] Nagaoka K et al 2015 Nucl. Fusion 55113020

[7] Ida K et al 2009 Phys. Plasmas 16056111 
[8] Yoshinuma M et al 2009 Nucl. Fusion 49062002

[9] Osakabe M et al 2014 Plasma Phys. Control. Fusion 56095011

[10] Takeiri Y et al 2017 Nucl. Fusion 57102023

[11] Goto M et al 2010 J. Phys. B: At. Mol. Opt. Phys. 43144023

[12] Huang X et al 2015 Plasma Fusion Res. 103402036

[13] Yoshinuma M et al 2010 Fusion Sci. Technol. 58375

[14] Du X D et al 2016 Nucl. Fusion 56016002

[15] Yokoyama M et al 2017 Nucl. Fusion 57126016

[16] Weynants R E et al 1999 Nucl. Fusion 391637

[17] Tokar M Z et al 1998 Nucl. Fusion 38961

[18] Tokar M Z et al 2000 Phys. Rev. Lett. 84895

[19] Dong J Q et al 1994 Phys. Rev. Lett. 13635

[20] Sudo S et al 2012 Rev. Sci. Instrum. 83023503

[21] Lunsford R et al 2017 Nucl. Fusion 57076008

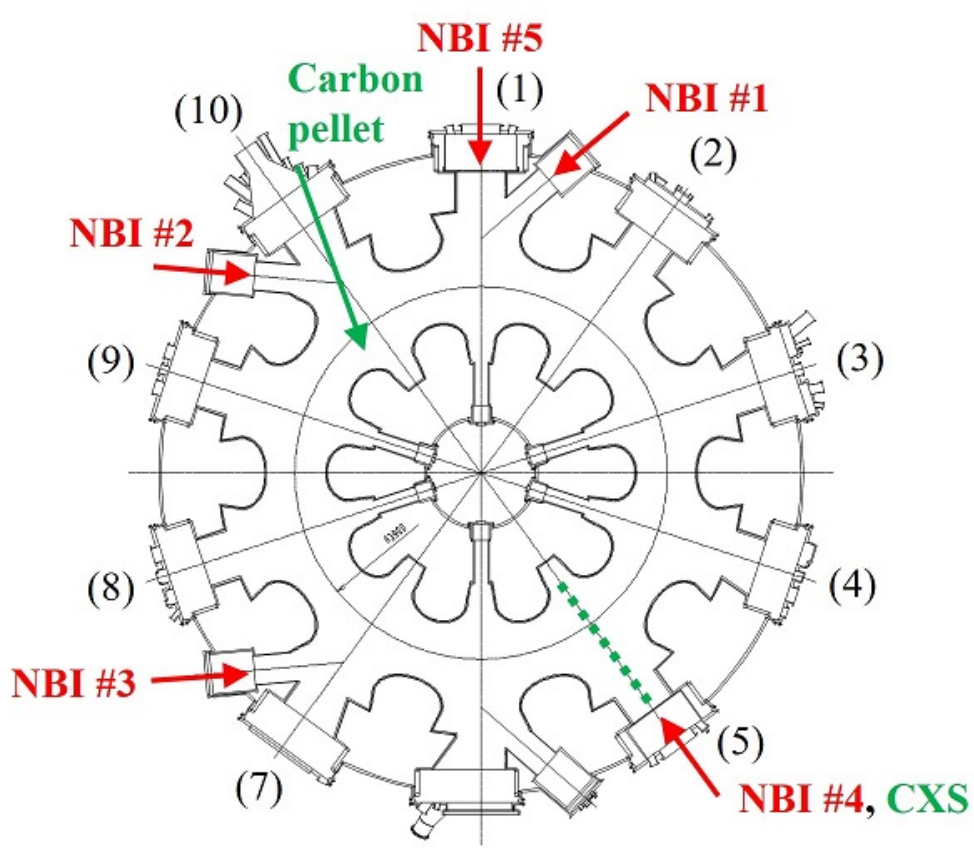

(6)

Figure 1. Experimental setup in LHD. 


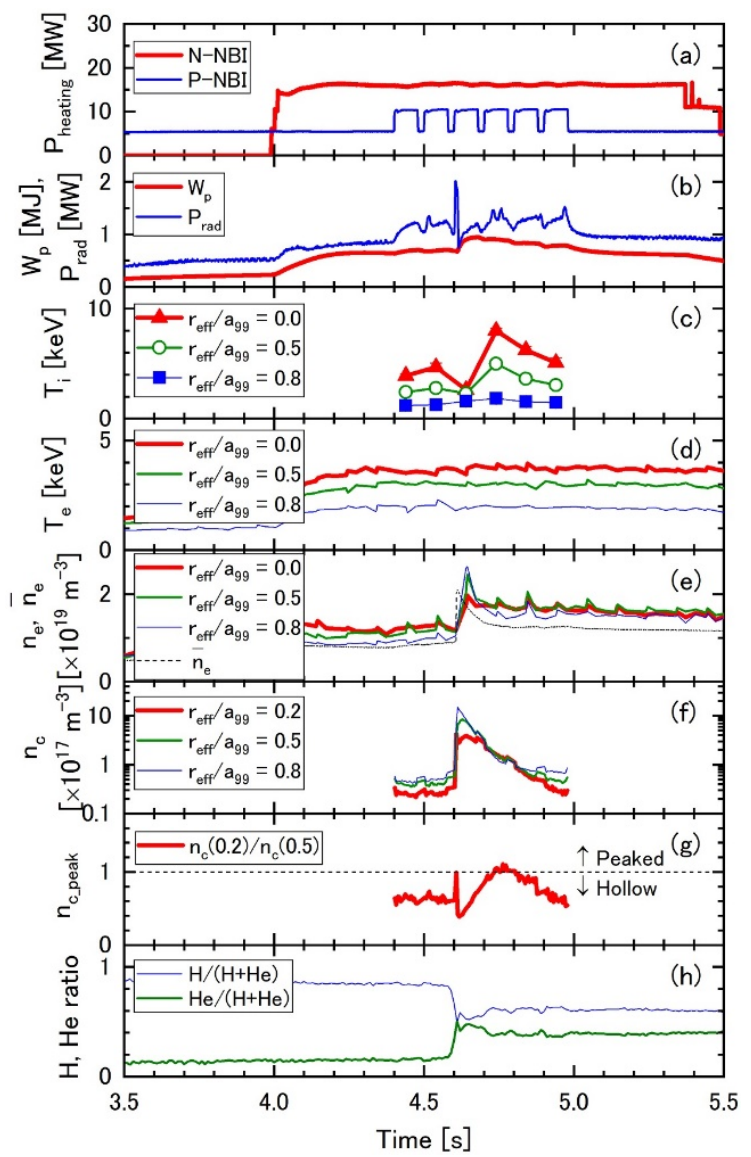

Figure 2. Time evolution in the hydrogen discharge (\#123145) of (a) heating power, (b) plasma stored energy and radiated power, (c) ion temperature, (d) electron temperature, (e) electron density and line averaged electron density, (f) carbon density, (g) peaking factor of carbon density profile, and (h) density fraction of hydrogen and helium. 


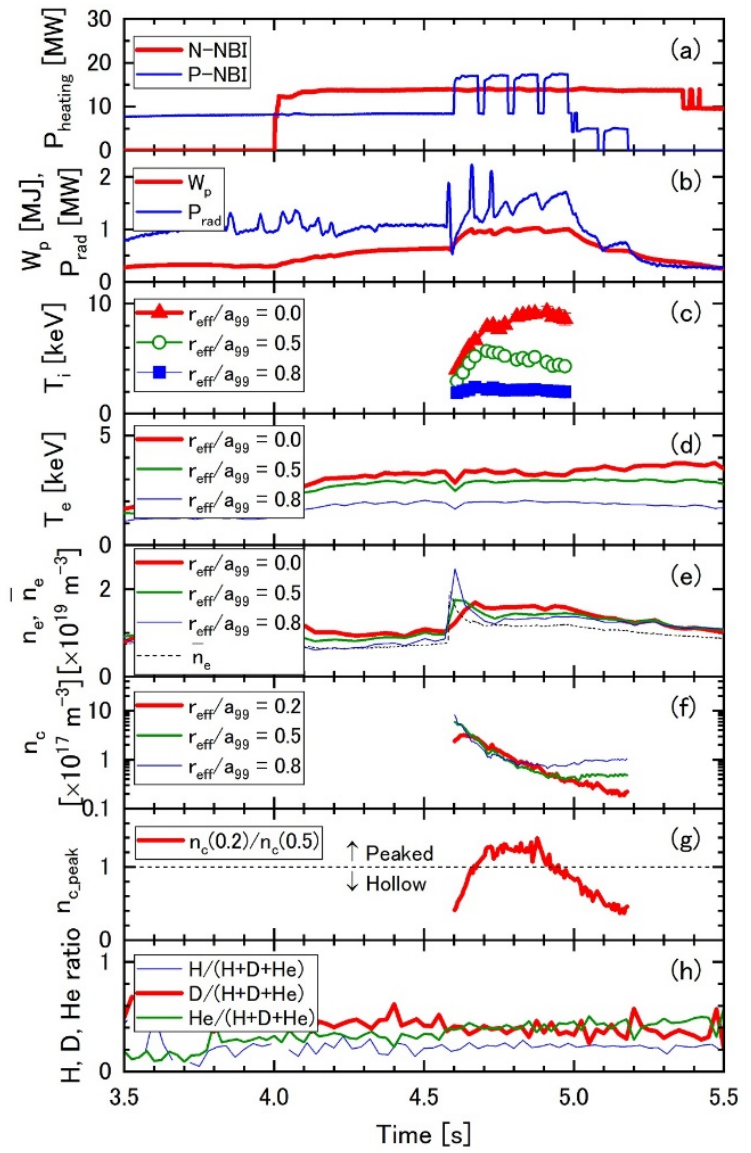

Figure 3. Time evolution in the deuterium discharge (\#133707) of (a) heating power, (b) plasma stored energy and radiated power, (c) ion temperature, (d) electron temperature, (e) electron density and line averaged electron density, (f) carbon density, (g) peaking factor of carbon density profile, and (h) density fraction of hydrogen, deuterium and helium.

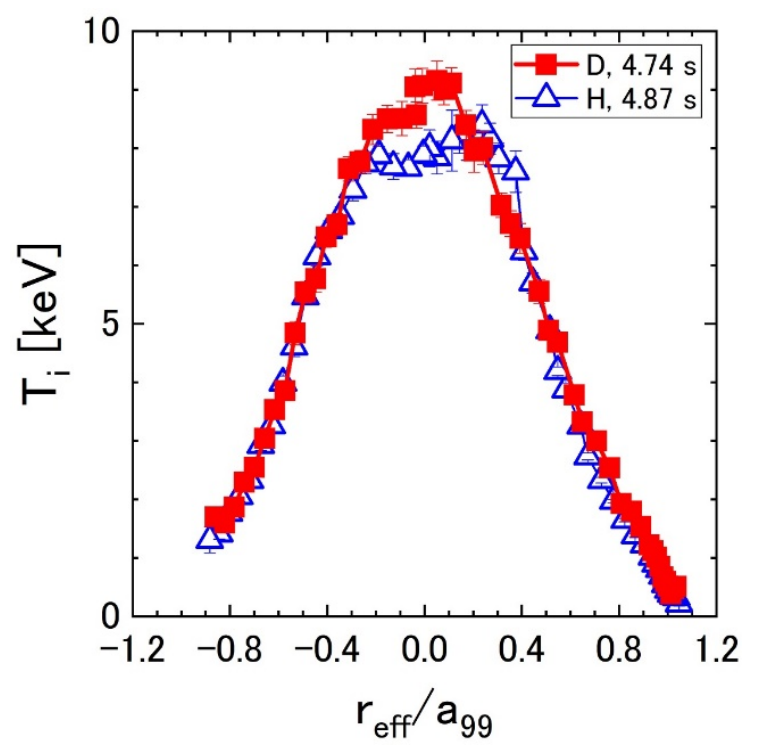


Figure 4. Ion temperature profiles in the deuterium (\#133707) and the hydrogen (\#123145) discharges at the time of the maximum central ion temperature.

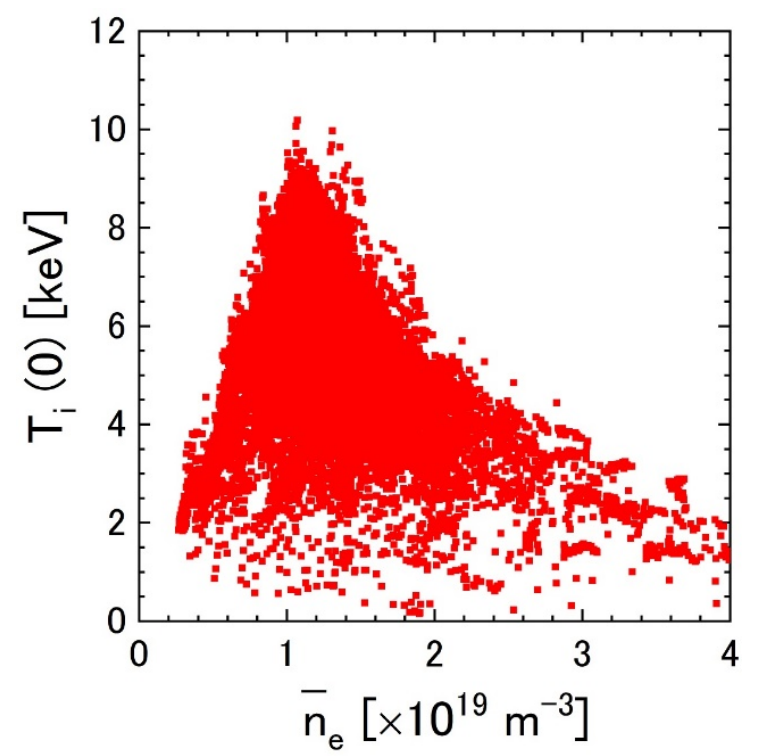

Figure 5. Line-averaged electron density dependence of central ion temperature in the all high- $T_{\mathrm{i}}$ deuterium discharges.
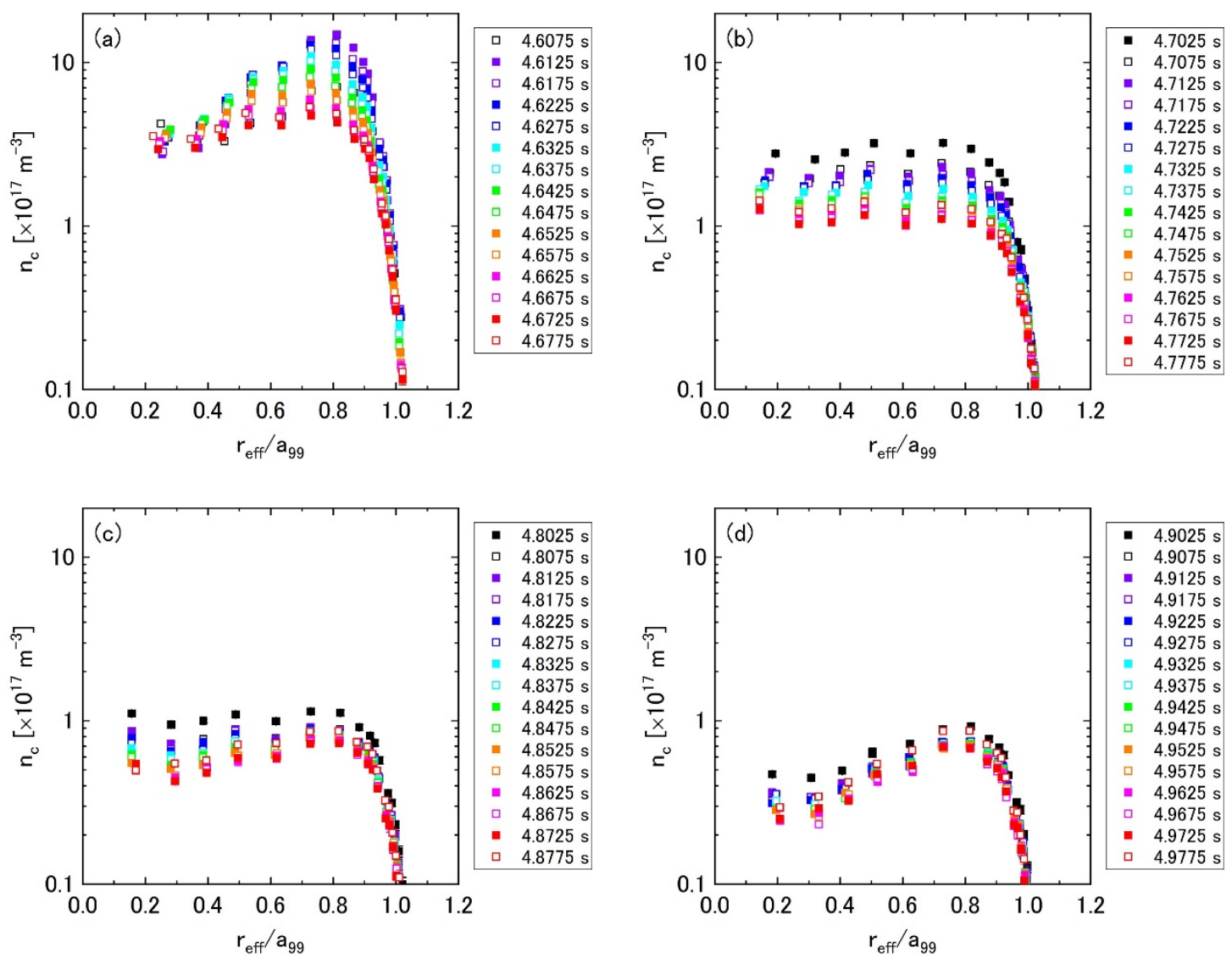
Figure 6. Time evolution of carbon density profiles in the hydrogen discharge (\#123145) after the injection of a carbon pellet.
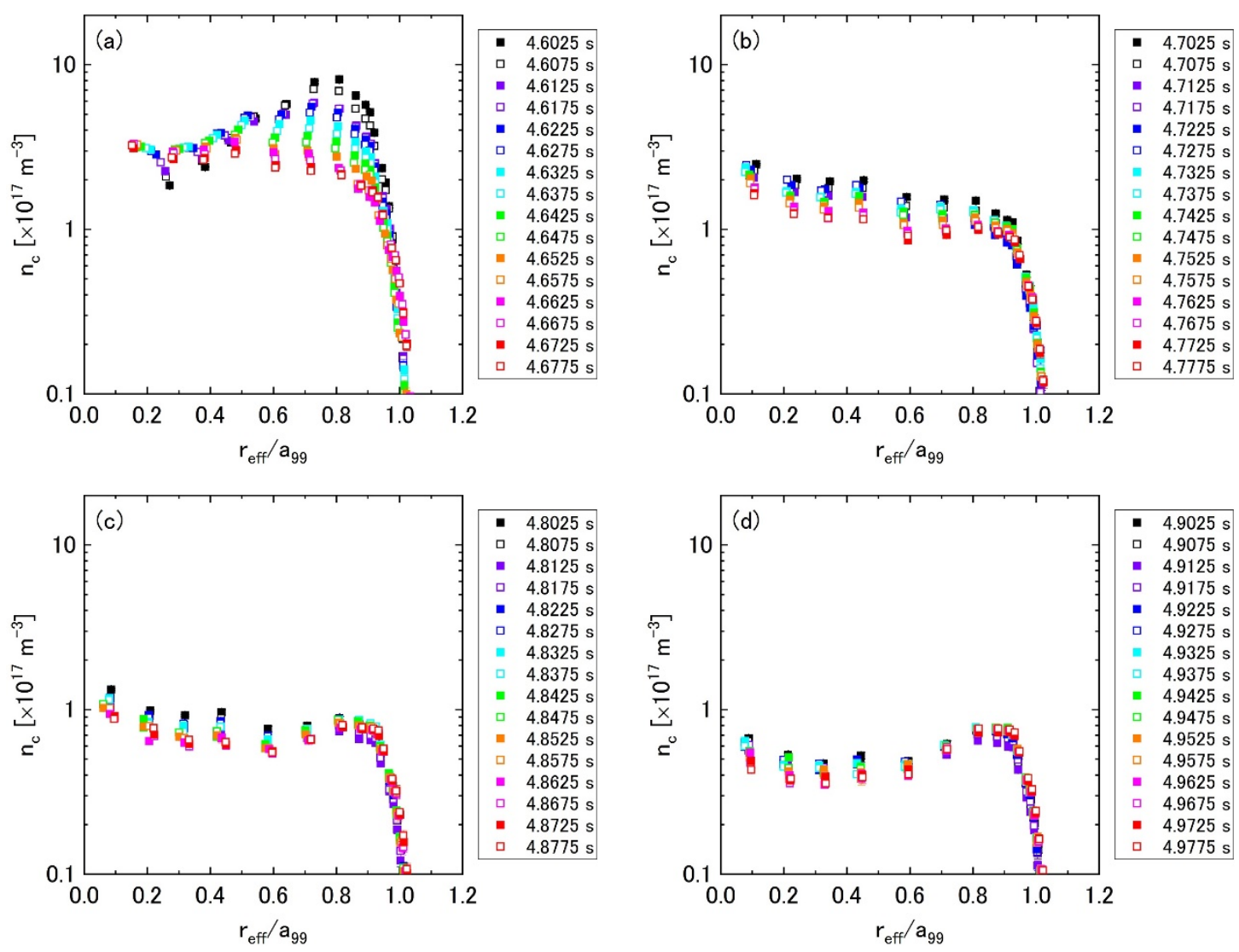

Figure 7. Time evolution of carbon density profiles in the deuterium discharge (\#133707) after the injection of a carbon pellet.
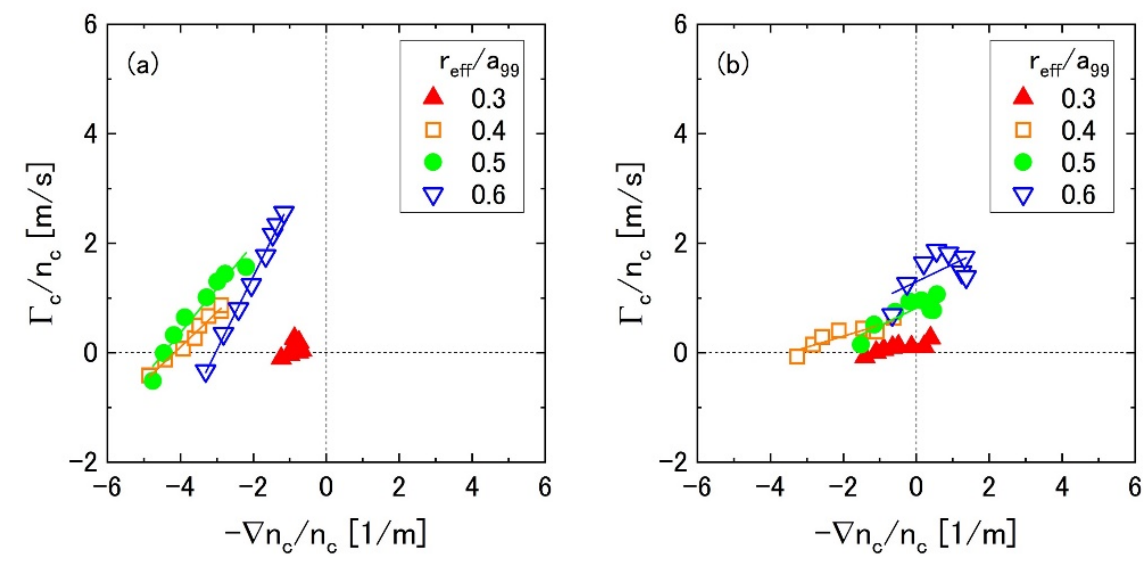

Figure 8. Carbon density gradient dependence on carbon particle flux normalized by carbon density in (a) the hydrogen plasma (\#123145) and (b) the deuterium plasma (\#133707). 


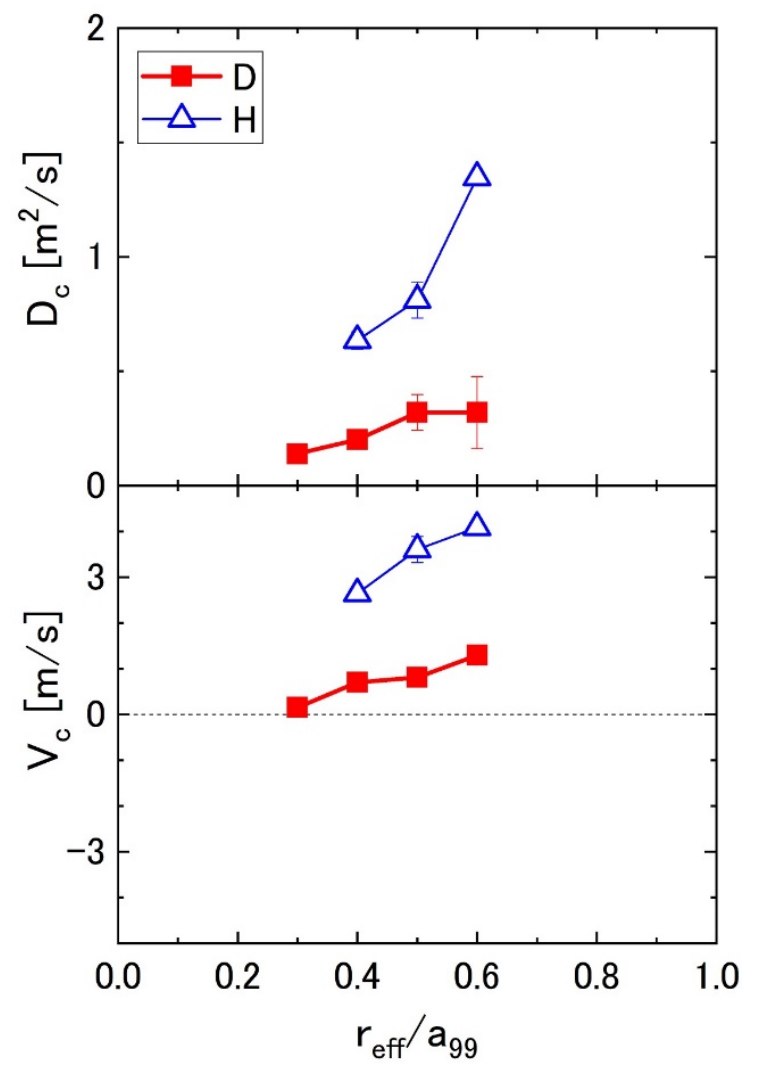

Figure 9. Radial profile of the diffusivity and convection velocity of the carbon density with comparison between the hydrogen plasma (\#123145) and the deuterium plasma (\#133707).
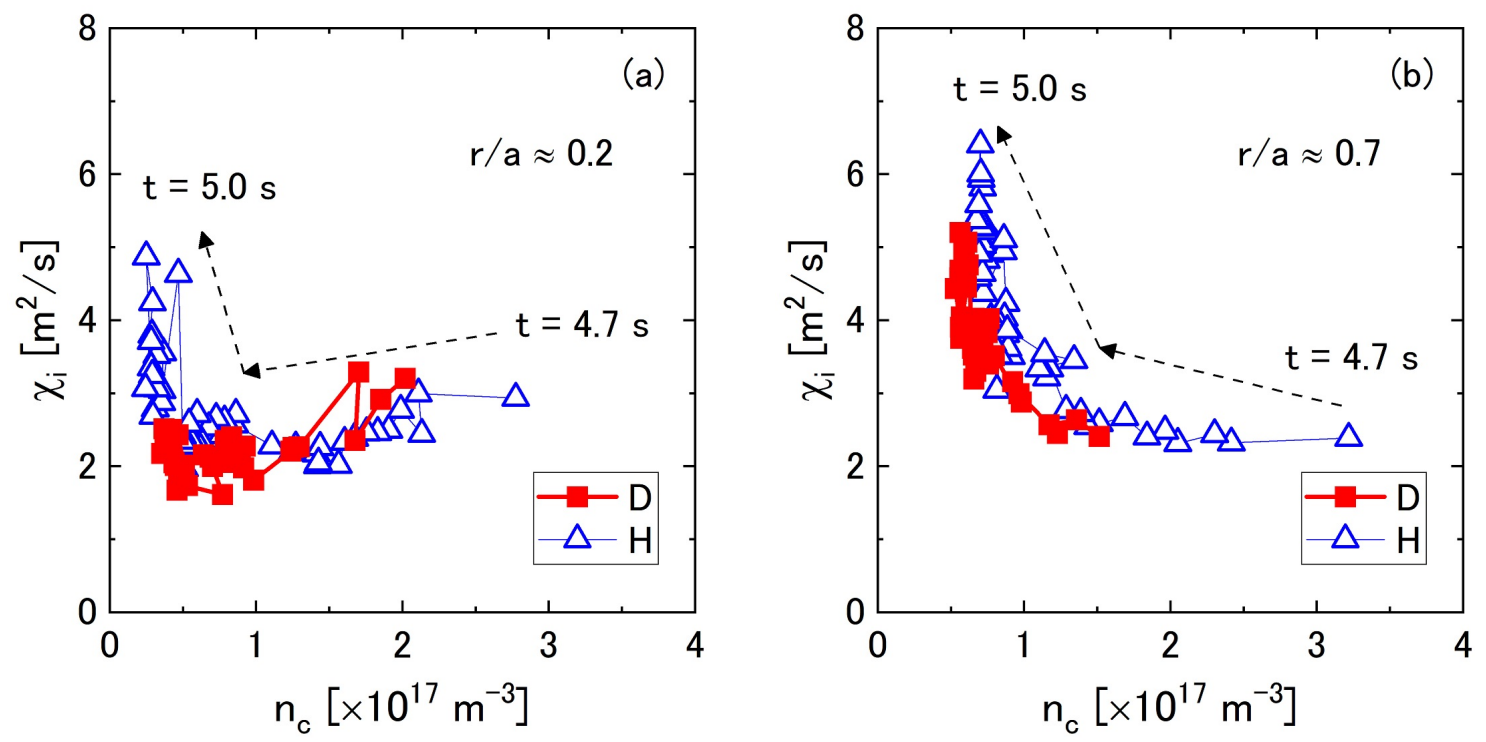

Figure 10. Carbon density dependence of ion thermal diffusivity as the comparison of hydrogen (\#123145) and deuterium (\#133707) discharges at (a) $r_{\text {eff }} / a_{99} \sim 0.2$ and (b) $r_{\text {eff }} / a_{99} \sim 0.7$. 


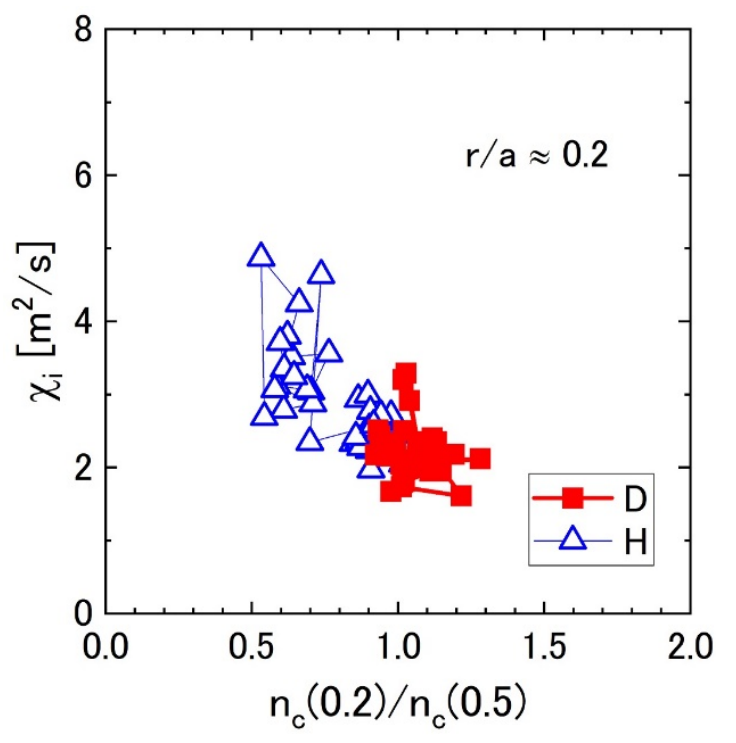

Figure 11. Carbon density peaking dependence of ion thermal diffusivity as the comparison of hydrogen (\#123145) and deuterium (\#133707) discharges at $r_{\mathrm{eff}} / a_{99} \sim 0.2$. 